\title{
Moderate intake of aspartame and sucralose with meals, but not fructose, does not exacerbate energy and glucose metabolism in estrogen- deficient rats
}

\author{
Jin Ah Ryuk, ${ }^{1}$ Suna Kang, ${ }^{2}$ James W. Daily, ${ }^{3}$ Byoung-Seob Ko ${ }^{1}$ and Sunmin Park ${ }^{2, *}$ \\ ${ }^{1}$ Korean Medicine Convergence Research Division, Korea Institute of Oriental Medicine, Daejeon, 305-811, South Korea \\ 2Department of Food and Nutrition, Obesity/Diabetes Research Center, Hoseo University, 165 Sechul-Ri, BaeBang-Yup, Asan-Si, ChungNam-Do, 336-795, South Korea \\ ${ }^{3}$ Department of R\&D, Daily Manufacturing Inc., Rockwell, NC, USA
}

(Received 16 February, 2019; Accepted 21 March, 2019; Published online 11 September, 2019)

Both nutritive and non-nutritive sweeteners may influence energy and glucose metabolism differently. The hypothesis that sucrose, fructose, aspartame, and sucralose intake differently modulate energy and glucose metabolism was tested in an estrogen-deficient animal model. At $\mathbf{3 0} \mathrm{min}$ after giving aspartame and sucralose (10 $\mathrm{mg} / \mathrm{kg}$ body weight), an oral glucose tolerance test (OGTT) was conducted with glucose, sucrose, and fructose in ovariectomized (OVX) rats. After OGTT, they were continuously fed high fat diets including either $10 \%$ corn starch (Control), $10 \%$ sucrose (Sucrose), $10 \%$ fructose (Fructose), $0.05 \%$ aspartame $+9.95 \%$ starch (Aspartame) or $0.05 \%$ sucralose $+9.95 \%$ starch (Sucralose) for 8 week. During $30 \mathrm{~min}$ after acute administration of aspartame and sucralose, serum glucose concentrations increased despite slightly increased serum insulin levels before glucose infusion. However, glucose tolerance was not significantly different among the groups. In chronic study, serum glucose concentrations were lowest and insulin highest at the overnight-fasted state in Aspartame and Sucralose. Postprandial serum glucagon-like peptide-1 (GLP-1) and insulin levels were higher in Aspartame and Sucralose than Control. Hepatic insulin signaling (pAkt $\rightarrow$ pGSK-3ß) and phosphoenolpyruvate carboxykinase (PEPCK) expression were lower in Sucralose and Aspartame than the Fructose. Serum acetate levels produced by gut microbiota were higher were lower in the fructose group than Aspartame and Sucralose groups. In conclusion, aspartame and sucralose with a meal might be preferable sweeteners to fructose and sucrose in estrogen deficient rats, and possibly post-menopausal women; however, this needs to be confirmed in human studies.

Key Words: non-nutritive sweeteners, fructose, GLP-1, glucose, insulin signaling

$\mathrm{O}$ besity increases the development of metabolic diseases such as type 2 diabetes, dyslipidemia, hypertension and stroke, ${ }^{(1)}$ and is associated with increased adipokines. ${ }^{(1)}$ After menopause, women develop central obesity which increases their susceptibility to metabolic diseases. Obesity is a major problem for menopausal women, but Asians are not as obese as Americans and Europeans. ${ }^{(2)}$ Asians easily develop hyperglycemia when insulin resistance increases due to obesity, since Asians have lower insulin secretion capacity than Caucasians. ${ }^{(3)}$ Consequently, Asians with the same body mass index (BMI) have a higher prevalence of metabolic diseases, especially type 2 diabetes, as Americans and Europeans. $^{(2,4)}$ Because Asians have lower thresholds of BMI and body fat mass for obesity related pathologies, obesity is defined at a lower BMI in many Asian countries.

Average total sucrose intake has increased from $20.6 \mathrm{~g}$ in 1993 to $72.1 \mathrm{~g}$ in 2013 , and average sucrose intake from processed foods increased from $10.1 \mathrm{~g}$ in 1993 to $44.7 \mathrm{~g}$ in 2013 in Korea. $34 \%$ of Koreans consume more than $50 \mathrm{~g}$ of sucrose daily from processed foods, which is a World Health Organization recommended limit. Among Korean children and adolescents, consumption of sucrose from sucrose-sweetened beverages was positively related to metabolic syndrome in the Korean Child-Adolescent Cohort Study. ${ }^{(5)}$ Moreover, the intakes of sucrose-sweetened and non-nutritive sweetener-sweetened beverages increase the risk of type 2 diabetes by $43 \%$ and by $21 \%$, respectively, compared to water intake, in post-menopausal women. ${ }^{(6)}$ Thus, the marked increase in consumption of nutritive and non-nutritive may be a factor in the increased incidence of diabetes.

Obesity is considered to be caused by an imbalance between energy consumption and expenditure, and sucrose-sweetened beverage intake is often blamed for obesity. However, after adjusting for energy intake and physical activity, an association between sucrose-sweetened beverage intake and obesity risk is inconsistent for children, adolescents, and adults. One prospective cohort study revealed a significant association between sucrosesweetened beverage intake and obesity risk in children ${ }^{(7)}$ while another one showed no significant association between sucrosesweetened beverage intake and BMI. ${ }^{(8)}$ A systematic review could not draw a conclusion about the causal relationship between sucrose-sweetened drinks intake and obesity in intervention, prospective and cross-sectional studies. ${ }^{(9)}$ Sucrose intake is also reported to be associated with insulin sensitivity and insulin secretion. ${ }^{(10)}$ A 12 -week treatment of $30 \%$ sucrose consumption increased weight gain and deteriorated glucose tolerance without changing serum insulin levels in mice. ${ }^{(11)}$ High intakes of sucrose and fructose, accompanied with high energy intake, increased fat deposition in the liver, adipose tissues and skeletal muscle, and elevated insulin resistance. ${ }^{(12-14)}$ However, it remains controversial. $^{(12)}$

Non-nutritive sweeteners have been used as substitutes for sucrose. Some non-nutritive sweeteners such as aspartame and sucralose have no calories but sweetness is much higher than sucrose. In previous large cohort studies, the intake of nonnutritive sweeteners decreased body weight and the risk of type 2 diabetes and coronary heart diseases. ${ }^{(15)}$ However, recent studies

To whom correspondence should be addressed.

E-mail: smpark@hoseo.edu 
have demonstrated that non-nutritive sweeteners stimulate adipogenesis and suppress lipolysis, ${ }^{(16)}$ but they do not influence the secretion of satiety hormones glucagon-like peptide-1 (GLP-1) and peptide YY or ghrelin from the intestines, gastrointestinal satiety peptides. ${ }^{(17)}$ Thus, the effects of sucrose and non-nutritive sweeteners on energy and glucose metabolism remain uncertain.

Most studies on the effects of sucrose and non-nutritive sweeteners on energy and glucose metabolism have been conducted by providing beverages to young animals and adolescents, but only a few studies have provided non-nutritive sweeteners in the meal instead of sucrose. Furthermore, post-menopausal women develop obesity with glucose and lipid dysregulation, and they often have high in sugar intake. ${ }^{(6,10)}$ Therefore, we hypothesized that sucrose, fructose and non-nutritive sweeteners (aspartame and sucralose) may differently influence energy and glucose metabolism, and we tested the hypothesis and explored the mechanism in an estrogen-deficient animal model.

\section{Materials and Methods}

Animal care. Female Sprague-Dawley rats aged about 10 weeks $(231 \pm 20 \mathrm{~g})$ were housed in individual stainless steel cages in a dedicated animal facility with temperature maintained at $23^{\circ} \mathrm{C}$ and a 12-h light/dark cycle. This study conformed with the Guide for the Care and Use of Laboratory Animals (8th edition, National Academies Press) and Use of Laboratory Animals and was approved by the Hoseo University Animal Care and Use Committee (HTRC-16-15). The rats obtained from DBL (Yeumsung-Kun, Korea) were allowed to acclimate for 1 week.

Ovariectomy operation. After anesthetization by subcutaneously injecting ketamine and xylazine mixture $(100$ and $10 \mathrm{mg} / \mathrm{kg}$ body weight) the rats had ovariectomy (OVX) as previously described. ${ }^{(18)}$ The OVX rats were then randomly divided into 5 groups of 12 each.

Experimental design. Fifty OVX rats were divided into 5 groups using a randomized block design. Each group was provided free access to water and an assigned diet (starch, sucrose, fructose, aspartame and sucralose). After 8 weeks of dietary intervention, the rats were fasted overnight and serum glucose concentrations were determined. Every week food intake was measured.

Acute oral glucose tolerance test. Prior to providing assigned diets, oral glucose tolerance test (OGTT) was conducted by orally giving $2 \mathrm{~g}$ of different sweet molecules per $\mathrm{kg}$ body weight, or by administering aspartame and sucralose $(10 \mathrm{mg} / \mathrm{kg}$ body weight) at doses with equivalent sweetness of $2 \mathrm{~g}$ glucose in OVX rats. Since sucrose and fructose themselves increase blood glucose levels, they were orally given instead of glucose. However, aspartame and sucralose do not increase blood glucose concentration itself although they may change serum insulin levels. At $30 \mathrm{~min}$ after oral intake of aspartame and sucralose the rats were administered $2 \mathrm{~g}$ glucose $/ \mathrm{kg}$ body weight and serum glucose and insulin levels were monitored. The solution containing sucrose, fructose or glucose was orally provided to the rats at $0 \mathrm{~min}$ for the rats of the Sucrose, Fructose and Glucose groups. Every week 2 rats from each group had an OGTT and then had a wash-out period for 6 days. In the following week rats had another OGTT with another sweet molecule. 10 rats were included in each group of glucose, sucrose, fructose, aspartame + glucose and sucralose + glucose. Serum glucose concentrations were measured at -30 , $-15,0$ and every $10 \mathrm{~min}$ until $120 \mathrm{~min}$ and serum insulin concentrations were determined at $-30,0,20,40$ and $90 \mathrm{~min}$. Serum GLP-1 levels were assayed at 0 and $60 \mathrm{~min}$ by GLP-1 ELISA kits (Crystal Chem, Elk Grove Village, IL).

Diet preparation. All groups were fed high fat diets [43 energy \% (En\%)] to exacerbate the energy, glucose and lipid metabolism after menopause as compared to diets low in fat..$^{(19-21)}$ Diet composition was 37\% from carbohydrate (10 En\% sucrose and $27 \mathrm{En} \%$ starch), $20 \mathrm{En} \%$ from protein (casein) and $43 \mathrm{En} \%$ from lard which was based on the modified AIN-93 formulation. ${ }^{(22)}$ To determine the effects of non-nutritive sweeteners and natural sweeteners on energy and glucose metabolism in OVX rats, $10 \mathrm{En} \%$ sucrose in the original modified AIN-93 formulation was switched to starch (10 En\% Control), fructose (10 En\% Fructose), sucrose (10 En\% Sucrose), starch (9.5 En\%) plus aspartame (0.05 En\% Aspartame), and starch (9.8 En\%) plus sucralose (0.05 En\% Sucralose). Since aspartame and sucralose have over 100-fold sweetness more than sucrose, they were used 200 times less than sucrose and the remaining portion was filled with starch.

Energy expenditure by indirect calorimetry. At the 7 th week of the assigned treatment, energy expenditure was assessed at the beginning of the dark phase of the light-dark cycle after $6 \mathrm{~h}$ of feed-deprivation. The rats were placed into metabolic chambers (airflow $=800 \mathrm{ml} / \mathrm{min}$ ) equipped with a computercontrolled $\mathrm{O}_{2}$ and $\mathrm{CO}_{2}$ measurement system (Biopac Systems Inc., Goleta, CA). The respiratory quotient (RQ) and resting energy expenditure (REE) were calculated using the equations provided by Niwa et al. ${ }^{(23)}$ Average oxygen consumption $\left(\mathrm{VO}_{2}\right)$ and average carbon dioxide production $\left(\mathrm{VCO}_{2}\right)$ were calculated using previously published methods and used to calculate carbohydrate and fat oxidation and the amount of oxygen consumed per gram of substrate oxidized. ${ }^{(24,25)}$

Glucose homeostasis and sample collection at the end of experiment. At the 7th week, blood was collected from overnight-fasted animals and at $30 \mathrm{~min}$ after assigned food intake. Serum was collected by centrifuging the blood at $3,000 \mathrm{~g}$ for $10 \mathrm{~min}$ and GLP-1 levels were measured by ELISA kit (Crystal Chem). At 2 days later the rats were subjected to an OGTT by oral administration of $2 \mathrm{~g}$ of glucose $/ \mathrm{kg}$ body weight. At $10 \mathrm{~min}$ intervals from 0 to $120 \mathrm{~min}$ post glucose loading, tail blood was collected for serum glucose measurements using a Glucose Analyzer II (Beckman, Palo Alto, CA). At 0, 20, 40, 90, and 120 min serum insulin concentrations were assessed using a ultrasensitive rat insulin ELISA kit (Crystal Chem). The trapezoidal rule was used for calculating means of the total area under the curve (AUC) for serum glucose and insulin concentrations.

Three days' post OGTT, the rats were anesthetized with the ketamine/xylazine as used earlier in the study and epididymal and retroperitoneal fat masses and uteri were excised and weighed. The uterus index, was calculated as uterus weight divided by body weight. Blood was collected from the portal vein and inferior vena cava for measuring short-chain fatty acids and other metabolic samples, respectively. Serum was prepared from the blood by centrifuging at 3,000 rpm for $20 \mathrm{~min}$. Human insulin $(1 \mathrm{U} / \mathrm{kg}$ body weight) was then injected into the inferior vena cava for determining hepatic insulin signaling. Serum and tissues were then stored at $-70^{\circ} \mathrm{C}$ for future use.

The homeostasis model assessment estimate (HOMA) for assessing insulin resistance (IR) and HOMA for insulin secretion (B) were calculated as previously reported. ${ }^{(22)}$ Serum $17 \beta$-estradiol levels were measured by ELISA kits (Enzo Life Sciences, NY). Serum triglyceride concentrations were measured by using colorimetry kits (Asan Pharmaceutical, Seoul, Korea).

Short-chain fatty acid analysis by gas chromatography. The solution of $n$-butanol:tetrahydrofuran:acetonitrile (50:30:20, $\mathrm{v}: \mathrm{v}: \mathrm{v})$ was mixed with serum with equal volume, and $5 \mathrm{M}$ HCL was added into the mixed solution. The mixture was vortexed for $5 \mathrm{~min}$ and centrifuged at $4^{\circ} \mathrm{C}, 3,000 \mathrm{~g}$ for $15 \mathrm{~min}$. The supernatant was taken and it was injected into Gas chromatography 680 (PerkinElmer Clarus, Waltham, MA) with Elite-FFAP column $(30 \mathrm{~m} \times 0.25 \mathrm{~mm} \times 0.25 \mu \mathrm{m})$. The carrier gas was helium and the flow rate was $1 \mathrm{ml} / \mathrm{min}$. The temperature was raised until $180^{\circ} \mathrm{C}$ at $10^{\circ} \mathrm{C} / \mathrm{min}$, and then the temperature was raised to $240^{\circ} \mathrm{C}$ at $20^{\circ} \mathrm{C} /$ min and retained for $6 \mathrm{~min}$. The inlet and detector temperature were $230^{\circ} \mathrm{C}$ and $250^{\circ} \mathrm{C}$, respectively. The flow rates of hydrogen, air, and helium were 45,450 , and $20 \mathrm{ml} / \mathrm{min}$, respectively. 
Immunoblot analysis. Livers were lysed in $20 \mathrm{mM}$ Tris buffer as previously reported.(19) Lysates equilibrated to equal amounts of protein $(30-50 \mu \mathrm{g})$ were immunoblotted with specific antibodies against protein kinase $\mathrm{B}$ (PKB/Akt), glycogen synthase kinase (GSK)-3 $\beta$, phosphoenol-pyruvate carboxykinase (PEPCK), and $\beta$-actin, and phosphorylated forms of $\mathrm{PKB}^{\mathrm{Ser} 473}$ and glycogen syntase kinase-3 $\beta$ (GSK-3 $\beta$ ) (Cell Signaling, Danvers, MA), as previously described. ${ }^{(19)}$ Intensities of protein expression were measured using Imagequant TL (Amersham Biosciences, Piscataway, NJ).

Statistical analysis. SAS software version 7 (SAS Institute, Cary, CA) was used for statistical analysis. Sample size was estimated using a $\mathrm{G}$ power program (power $=0.90$ and effect size $=$ 0.5 ) and a sample size of 10 per group was required. When the results were normally distributed as confirmed by using Proc univariate, results are given as means \pm SD. Variables spanning multiple time points were analyzed using two-way repeated measures analysis of variance (ANOVA), with independent variables being time and group and the interaction term being between time and group. Measurements were statistically analyzed by one-way ANOVA. Significance of differences among the multiple groups was assessed by Tukey's test at the level of $p<0.05$.

\section{Results}

Acute OGTT. At $30 \mathrm{~min}$ after aspartame and sucralose administration, serum glucose concentrations had increased about 6-8 mg/dl compared to saline treatments in OVX rats. Since aspartame and sucralose do not include energy sources, $2 \mathrm{~g}$ glucose per $\mathrm{kg}$ body weight was orally given. Sucrose or fructose ( $2 \mathrm{~g}$ per $\mathrm{kg}$ body weight) was orally provided instead of glucose. Serum glucose levels increased until 30-40 min after glucose, sucrose or fructose provision and the levels were lowered after the peak (Fig. 1A). The peak levels were lowest in the fructose group among the groups and the levels were lower in sucrose group in the second part of the OGTT (Fig. 1A). The concentrations were similar in glucose administered group with or without aspartame and sucralose uptake. After the peak serum glucose levels were lowered in all groups. Sucrose quickly decreased serum glucose levels than the glucose and glucose plus aspartame or sucralose. Aspartame and sucralose consumption did not affect serum glucose levels in the 2nd part of OGTT (Fig. 1B). Area under the curve (AUC) during entire OGTT was highest in the Glucose group among all groups and the AUC was lower in the Aspartame group than the Glucose slight but significantly $(p=0.048)$.
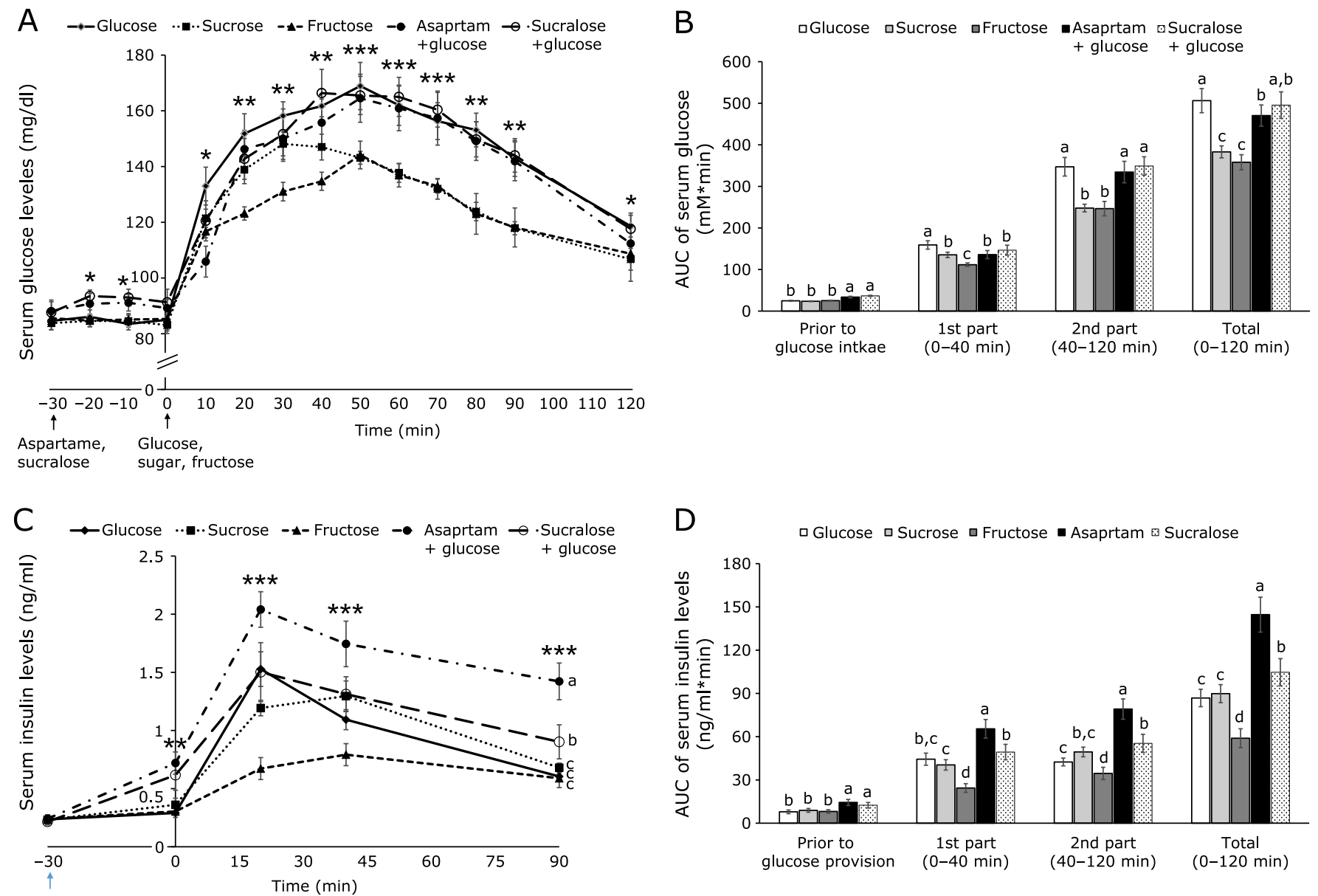

Fig. 1. Serum glucose and insulin levels after oral infusion of glucose, sucrose or fructose prior to the oral intake of distilled water, aspartame, or sucralose. Rats had $10 \mathrm{mg}$ aspartame, sucralose or distilled water per $\mathrm{kg}$ body weight and at 30 min later, the rats had $2 \mathrm{~g}$ glucose, fructose or sucrose per kg body weight. Rats in the Aspartame and Sucralose groups had glucose and those in the Sucrose and Fructose had sucrose and fructose, respectively. Serum glucose levels were measured every $10 \mathrm{~min}$ after giving aspartame, sucralose and distilled water (-30 min) (A). Area under the curve (AUC) of serum glucose levels was calculated in 3 parts (B): prior to oral glucose infusion ( $-30-0$ min), 1st part after glucose infusion (0-40 $\mathrm{min})$ and $2 \mathrm{nd}$ part after glucose infusion (40-120 min). Serum insulin levels were measured at $-30,0,20,40$, and $90 \mathrm{~min}(\mathrm{C})$. AUC of serum insulin levels were assayed in the 1 st part after glucose infusion ( $0-20 \mathrm{~min})$ and 2 nd part after glucose infusion (20-40 min) (D). The dots and bars represent means $\pm \operatorname{SD}(n=12)$. *Significantly different among all groups in one-way ANOVA at $p<0.05, * *$ at $p<0.01, * * *$ at $p<0.001$. ${ }^{a}, \mathrm{~b}, \mathrm{CT}$ (he different letters on the bars represent significant differences among the groups by Tukey's test at $p<0.05$. 
Sucrose and Fructose groups were lower than the Aspartame group (Fig. 1B).

Serum insulin levels were slightly elevated at $30 \mathrm{~min}$ after aspartame and sucralose were given in comparison to the saline groups in OVX rats (Fig. 1C). Glucose or sucrose provision increased serum insulin levels at $20 \mathrm{~min}$ and then the levels were lowered. Glucose with aspartame increased serum insulin levels the most at 20 min among the groups. However, fructose increased serum insulin levels much less than other groups at $20 \mathrm{~min}$, but remained elevated until at least $45 \mathrm{~min}$ (Fig. 1C). Sucrose was digested into glucose and fructose and the serum insulin levels were in between of glucose and fructose administration (Fig. 1C). Even though serum glucose levels in the Aspartame and Sucralose groups were similar to the Glucose group, serum insulin levels were much higher in the Aspartame and Sucralose groups during entire OGTT (Fig. 1C). Serum insulin levels were highest in the Aspartame among the groups.

AUC of serum insulin concentrations were higher in the aspartame and sucralose groups than the other groups for $30 \mathrm{~min}$ after aspartame and sucralose intake and remained elevated in the Aspartame group 30 min longer than in the sucralose group (Fig. 1D). After $2 \mathrm{~g} / \mathrm{kg}$ body weight glucose, fructose or sucrose intake AUC in the 1st part was higher in the ascending order of fructose, sucrose, glucose, sucralose and aspartame. The AUC in the 2nd part also showed a similar pattern to that of the 1st part (Fig. 1D). AUC of serum insulin during entire OGTT were elevated with aspartame and sucralose administration with glucose compared to glucose administration only (Fig. 1D). Fructose administration lowered the AUC of serum insulin levels than glucose administration (Fig. 1D). The results indicated that in acute administration of artificial sweetener and glucose, aspartame and sucralose treatment increased insulin resistance and aspartame elevated it more than Sucralose during OGTT.
Energy metabolism and short-chain fatty acids in the circulation. Final body weight was not significantly different among the different interventions. Weight gain during experimental periods tended to be higher in the Fructose group than the other groups but it was not significantly different $(p=0.07$; Table 1). However, uterine fat mass was much higher in the Fructose group than in the other groups whereas retroperitoneal fat mass was lowered in the descending order of Fructose, Sucrose, Control, Aspartame and Sucralose (Table 1). Visceral fat mass, the sum of uterine fat and retroperitoneal fat mass, was highest in the Fructose group among all groups and it was higher in the Sucrose and Control groups than the Aspartame and Sucralose groups (Table 1). Uterus weight and serum 17 $\beta$-estradiol levels were not significantly different among all the groups (Table 1).

Among short-chain fatty acids serum acetate levels were higher only in the Fructose compared to the Control (Table 1). By contrast, serum propionate and butyrate levels were not significantly different among the groups. They tended to be lower in the Fructose than the Control (Table 1).

Food intake was not significantly different among the groups (Table 2). Energy expenditure was lower in the Fructose group than in the Control group (Table 2). Oxygen consumption was lower in the Fructose group than the other groups. RQ was not significantly different among the groups. Carbohydrate oxidation was lowered in the descending order of Aspartame, Sucralose, Control, Sucrose, and Fructose (Table 2). Fat oxidation was opposite to carbohydrate oxidation (Table 2). Thus, the greater weight gain in the Fructose group was associated with lower energy expenditure, not higher energy intake. Aspartame and sucralose did not alter carbohydrate and fat oxidation compared to the Control (Table 2).

Glucose metabolism. Serum glucose concentrations at the overnight fasting state decreased in the descending order of the

Table 1. Body weight, visceral fat mass and uterine weight

\begin{tabular}{lccccc}
\hline & $\begin{array}{c}\text { Control } \\
(n=12)\end{array}$ & $\begin{array}{c}\text { Sucrose } \\
(n=12)\end{array}$ & $\begin{array}{c}\text { Fructose } \\
(n=12)\end{array}$ & $\begin{array}{c}\text { Aspartame } \\
(n=12)\end{array}$ & $\begin{array}{c}\text { Sucralose } \\
(n=12)\end{array}$ \\
\hline Final body weigt $(\mathrm{g})$ & $301 \pm 25$ & $300 \pm 26$ & $309 \pm 27$ & $303 \pm 21$ & $303 \pm 19$ \\
Body weight gain (g/8 weeks) & $102 \pm 9.3$ & $102 \pm 8.8$ & $111 \pm 10.7$ & $105 \pm 9.2$ & $101 \pm 9.7$ \\
Uterine fat mass (g) & $4.70 \pm 0.87^{\mathrm{b}}$ & $5.04 \pm 0.51^{\mathrm{b}}$ & $7.31 \pm 0.97^{\mathrm{a}}$ & $4.64 \pm 0.65^{\mathrm{b}}$ & $4.94 \pm 0.68^{\mathrm{b}}$ \\
Retroperitoneal fat mass $(\mathrm{g})$ & $3.78 \pm 0.76^{\mathrm{b}}$ & $4.24 \pm 0.65^{\mathrm{b}}$ & $6.16 \pm 0.98^{\mathrm{a}}$ & $3.29 \pm 0.54^{\mathrm{c}}$ & $3.20 \pm 0.52^{\mathrm{c}}$ \\
Visceral fat mass $(\mathrm{g})$ & $8.6 \pm 1.7^{\mathrm{c}}$ & $10.6 \pm 1.9^{\mathrm{b}}$ & $13.5 \pm 2.0^{\mathrm{a}}$ & $7.7 \pm 1.0^{\mathrm{c}}$ & $8.1 \pm 1.1^{\mathrm{c}}$ \\
Uterus weight $(\mathrm{g})$ & $0.25 \pm 0.05$ & $0.24 \pm 0.06$ & $0.25 \pm 0.05$ & $0.26 \pm 0.05$ & $0.25 \pm 0.06$ \\
Serum acetate $(\mu \mathrm{M})$ & $165 \pm 18^{\mathrm{a}}$ & $167 \pm 20^{\mathrm{a}}$ & $192 \pm 21^{\mathrm{b}}$ & $172 \pm 19^{\mathrm{a}}$ & $169 \pm 19^{\mathrm{a}}$ \\
Serum propionate $(\mu \mathrm{M})$ & $54.6 \pm 5.7$ & $53.1 \pm 4.8$ & $51.4 \pm 5.9$ & $55.5 \pm 5.2$ & $53.9 \pm 5.6$ \\
Serum butyrate $(\mu \mathrm{M})$ & $24.6 \pm 2.0$ & $23.5 \pm 1.7$ & $22.7 \pm 2.1$ & $23.2 \pm 1.9$ & $23.6 \pm 1.8$ \\
\hline
\end{tabular}

The ovariectomized (OVX) rats were provided with a $45 \%$ fat diet with $10 \%$ starch, sucrose, fructose, aspartame + starch and sucralose + starch in a respective group for 8 weeks. Visceral fat mass was calculated by sum of uterine fat mass and retroperitoneal fat mass. ${ }^{a}, \mathrm{~b}, \mathrm{c}, \mathrm{The}$ different letters in the means represent significant differences among the groups by Tukey's test at $p<0.05$.

Table 2. Energy metabolism

\begin{tabular}{|c|c|c|c|c|c|}
\hline & $\begin{array}{l}\text { Control } \\
(n=12)\end{array}$ & $\begin{array}{l}\text { Sucrose } \\
(n=12)\end{array}$ & $\begin{array}{c}\text { Fructose } \\
(n=12)\end{array}$ & $\begin{array}{l}\text { Aspartame } \\
(n=12)\end{array}$ & $\begin{array}{c}\text { Sucralose } \\
(n=12)\end{array}$ \\
\hline Food intake (g/day) & $15.1 \pm 1.2$ & $14.7 \pm 1.2$ & $14.5 \pm 1.1$ & $15.1 \pm 1.2$ & $15.8 \pm 1.7$ \\
\hline Energy expenditure $\left(\mathrm{ml} \cdot \mathrm{kg}^{-0.75} \cdot \mathrm{min}^{-1}\right)$ & $126 \pm 13^{a}$ & $119 \pm 13^{a, b}$ & $107 \pm 13^{b}$ & $131 \pm 14^{a}$ & $129 \pm 13^{a}$ \\
\hline $\mathrm{VO}_{2}\left(\mathrm{ml} \cdot \mathrm{kg}^{-0.75} \cdot \mathrm{min}^{-1}\right)$ & $17.7 \pm 1.8^{a}$ & $17.0 \pm 1.7^{\mathrm{a}, \mathrm{b}}$ & $15.3 \pm 1.6^{b}$ & $18.4 \pm 1.7^{a}$ & $18.4 \pm 1.8^{a}$ \\
\hline Respiratory quotient & $0.85 \pm 0.07$ & $0.85 \pm 0.07$ & $0.85 \pm 0.08$ & $0.86 \pm 0.07$ & $0.85 \pm 0.08$ \\
\hline Carbohydrate oxidation $\left(\mathrm{mg} \cdot \mathrm{kg}^{-0.75} \cdot \mathrm{min}^{-1}\right)$ & $6.5 \pm 0.7^{a}$ & $6.2 \pm 0.7^{a, b}$ & $5.6 \pm 0.6^{b}$ & $7.1 \pm 0.8^{\mathrm{a}}$ & $6.7 \pm 0.7^{a}$ \\
\hline Fat oxidation $\left(\mathrm{mg} \cdot \mathrm{kg}^{-0.75} \cdot \mathrm{min}^{-1}\right)$ & $6.9 \pm 0.7^{a}$ & $6.5 \pm 0.7^{a, b}$ & $5.8 \pm 0.8^{\mathrm{b}}$ & $6.6 \pm 0.6^{a}$ & $7.0 \pm 1.0^{\mathrm{a}}$ \\
\hline
\end{tabular}

The ovariectomized (OVX) rats were provided with a $45 \%$ fat diet with $10 \%$ starch, sucrose, fructose, aspartame + starch, and sucralose + starch in a respective group for 7 weeks. After $16 \mathrm{~h}$ fasting, energy expenditure was measured by indirect calorimeter. a,b,cThe different letters in the means represent significant differences among the groups by Tukey's test at $p<0.05$. 
Fructose, Sucrose, Aspartame, Control and Sucralose (Table 3). Serum insulin concentrations at the overnight fasted state showed an opposite trend to serum glucose concentrations. Serum insulin levels were lowest in the Fructose group and the levels were highest in the Aspartame group among the groups (Table 3). HOMA-IR, an index of insulin resistance, was highest in Sucrose and Aspartame groups among the groups and it was higher in the Fructose and Sucralose than the Control. HOMA for insulin secretion (HOMA-B), an index of insulin secretion, was elevated in the ascending order of Aspartame $=$ Sucralose, Control, Sucrose, and Fructose (Table 3). Thus, sucalose might be preferable to fructose and sucrose in glucose metabolism.
After the glucose challenge, serum glucose concentrations in all groups increased until 20-40 min and then they gradually decreased (Fig. 2A). The peak levels were markedly higher in the Fructose and Sucrose groups than the other groups (Fig. 2A). Serum glucose concentrations markedly decreased after the peak at $20 \mathrm{~min}$ in the Fructose group but the concentrations in the Sucrose group were maintained at the peak levels until $50 \mathrm{~min}$. Serum glucose concentrations at the peak were much lower in the Control, Aspartame and Sucralose groups than the Sucrose and Fructose groups (Fig. 2A).

The AUC of serum glucose levels at the 1st part was much higher in the Sucrose and Fructose groups than the other groups

Table 3. Glucose metabolism in overnight-fasted states

\begin{tabular}{|c|c|c|c|c|c|}
\hline & $\begin{array}{l}\text { Control } \\
(n=12)\end{array}$ & $\begin{array}{l}\text { Sucrose } \\
(n=12)\end{array}$ & $\begin{array}{c}\text { Fructose } \\
(n=12)\end{array}$ & $\begin{array}{l}\text { Aspartame } \\
(n=12)\end{array}$ & $\begin{array}{l}\text { Sucralose } \\
(n=12)\end{array}$ \\
\hline Serum glucose (mg/dl) & $89.1 \pm 5.2^{c}$ & $109 \pm 5.5^{b}$ & $118.2 \pm 8.2^{\mathrm{a}}$ & $90.0 \pm 4.4^{c}$ & $86.7 \pm 5.4^{c}$ \\
\hline Serum insulin $(\mathrm{ng} / \mathrm{ml})$ & $1.22 \pm 0.23^{b, c}$ & $1.29 \pm 0.21^{b}$ & $1.07 \pm 0.18^{c}$ & $1.54 \pm 0.27^{\mathrm{a}}$ & $1.43 \pm 0.23^{\mathrm{a}, \mathrm{b}}$ \\
\hline HOMA-IR & $6.0 \pm 0.7^{c}$ & $7.8 \pm 0.8^{\mathrm{a}}$ & $7.0 \pm 0.7^{b}$ & $7.7 \pm 0.8^{\mathrm{a}}$ & $6.9 \pm 0.8^{\mathrm{b}}$ \\
\hline HOMA-B & $371 \pm 43^{b}$ & $321 \pm 45^{c}$ & $246 \pm 29^{d}$ & $463 \pm 46^{\mathrm{a}}$ & $447 \pm 54^{a}$ \\
\hline Serum triglyceride $(\mathrm{mg} / \mathrm{dl})$ & $117 \pm 13^{b}$ & $109 \pm 8.6^{b, c}$ & $138 \pm 14^{a}$ & $97.8 \pm 5.9^{c}$ & $104 \pm 6.7^{c}$ \\
\hline
\end{tabular}

The ovariectomized (OVX) rats were provided with a $45 \%$ fat diet with $10 \%$ starch, sucrose, fructose, aspartame + starch, and sucralose + starch in a respective group for 8 weeks. HOMA-IR, homeostatic Model Assessment for Insulin Resistance; HOMA-B, HOMA for insulin secretion. a,b,cThe different letters in the means represent significant differences among the groups by Tukey's test at $p<0.05$.
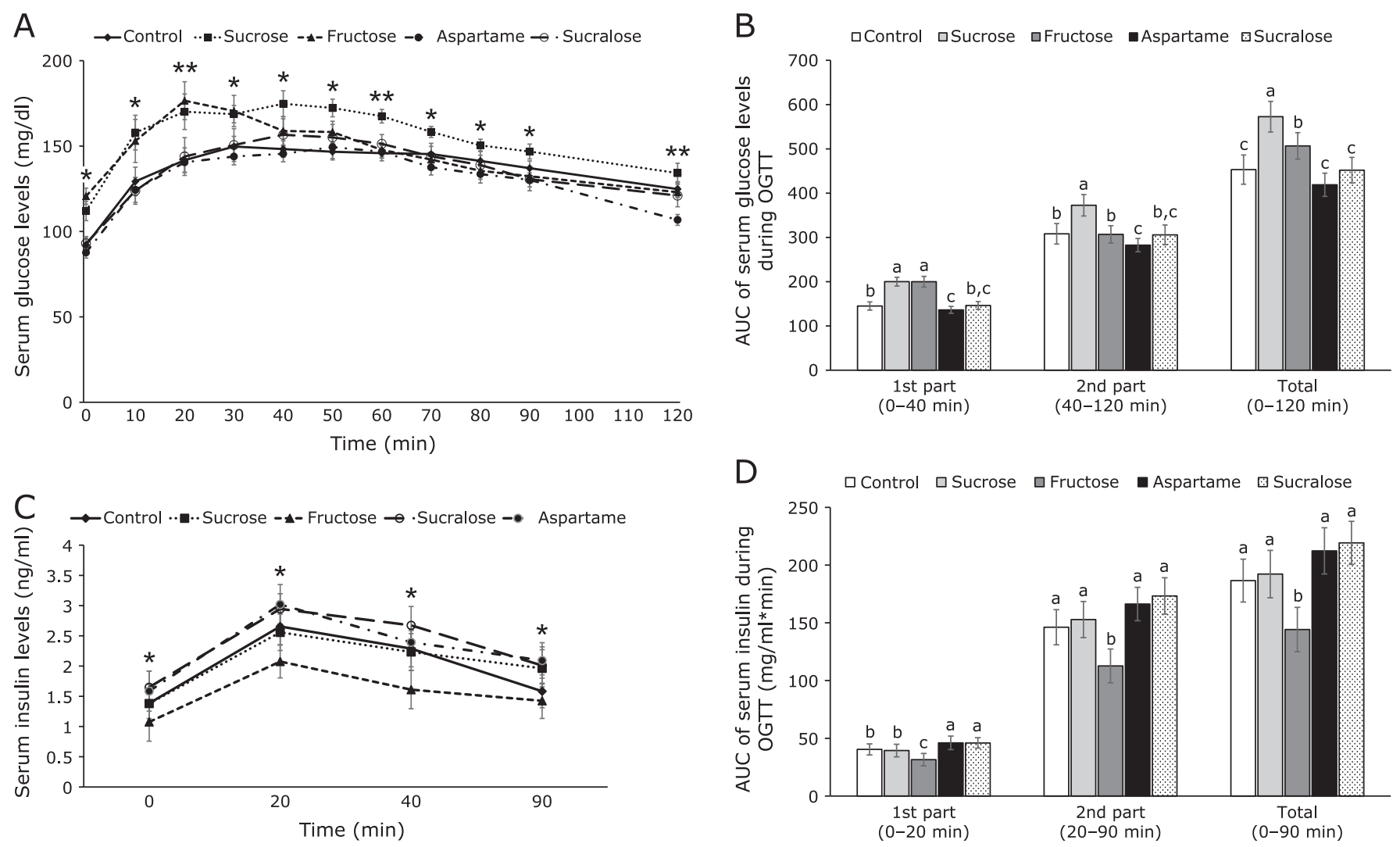

Fig. 2. The changes of serum glucose levels and areas under the curve of glucose and insulin during the oral glucose tolerance test (OGTT) after 7 week consumption of the assigned sweeteners. The ovariectomized (OVX) rats were provided with a $45 \%$ fat diet with $10 \%$ starch, sucrose, fructose, aspartame + starch and sucralose + starch for 8 weeks. At the 7th week, $2 \mathrm{~g}$ of glucose/kg body weight was orally administered and the serum glucose and insulin levels were measured at the indicated times. The changes in the serum glucose (A) and insulin (B) levels were measured during the OGTT. The average of the area under the curve (AUC) of glucose (C) and insulin (D) during the first part (0-40 min) and second part (40$120 \mathrm{~min})$ of the OGTT. The dots and bars represent means \pm SD $(n=12)$. *Significantly different among all groups in one-way ANOVA at $p<0.05$, ** at $p<0.01$. ${ }^{a}, \mathrm{~b}, \mathrm{c}$ The different letters on the bars represent significant differences among the groups by Tukey's test at $p<0.05$. 
whereas the AUC in the Aspartame group was lower than the Control (Fig. 2B). The AUC of serum glucose levels at the 2nd part was biggest in the Sucrose group among all the groups and that was smallest in the Aspartame group (Fig. 2B).

The changes in serum glucose concentrations during OGTT were associated with insulin secretion and insulin resistance. Serum insulin concentrations were elevated until $20 \mathrm{~min}$ and

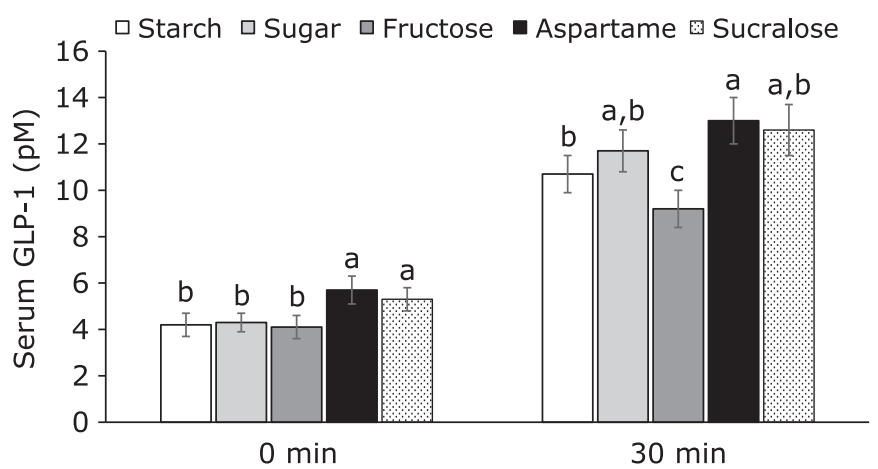

Fig. 3. Serum GLP-1 levels at $30 \mathrm{~min}$ after assigned meal intake. After 7 weeks of feeding with a $45 \%$ fat diet with $10 \%$ starch, sucrose, fructose, aspartame + starch and sucralose + starch in the ovariectomized (OVX) rats, the rats had blood collection in overnight fasting state and $1 \mathrm{~h}$ food provision. Serum GLP-1 levels were measured from the blood. The bars represent means \pm SD $(n=12)$. ${ }^{a}, b, c$ The different letters on the bars represent significant differences among the groups by Tukey's test at $p<0.05$.

A

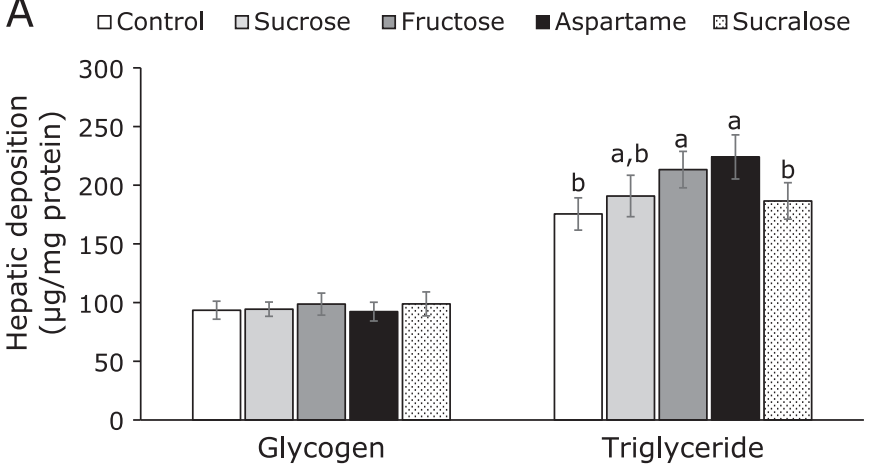

C

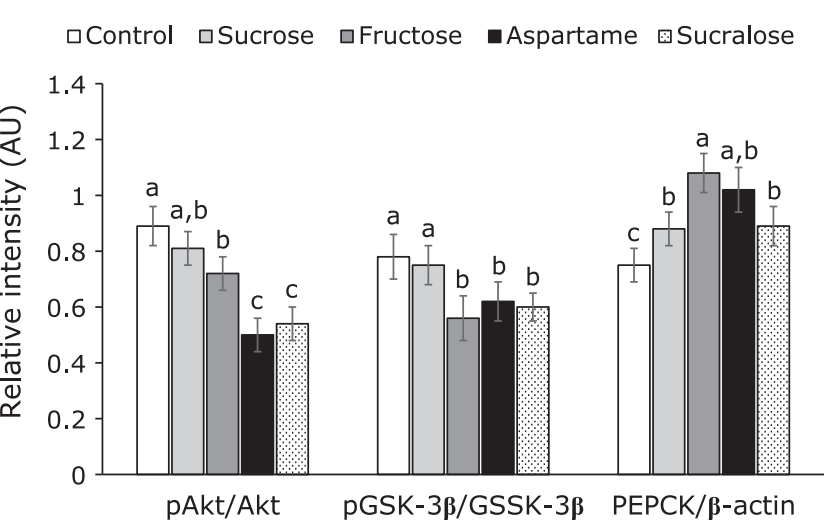

decreased during OGTT in the rats of all groups (Fig. 2C). The peak serum insulin levels at $20 \mathrm{~min}$ were higher in the ascending order of the Fructose, Sucrose and Control, Aspartame, and Sucralose groups (Fig. 2C). Serum insulin concentrations declined after the peak levels in different patterns. The AUC of serum insulin concentrations at the 1 st part (0-20 min) during OGTT was lowest in the Fructose group and it was higher in the non-nutritive sweetener groups than the Control and Sucrose groups (Fig. 2D). The AUC of the 2nd part (20-90 min) was lowest in Fructose among the groups and the AUC was not significantly different among the other groups (Fig. 2D). These results indicated that insulin resistance during hyperglycemia was higher in the Sucralose than the Fructose group.

After providing food for $30 \mathrm{~min}$, serum GLP-1 levels were higher in the Aspartame and Sucralose groups than the other groups at $0 \mathrm{~min}$ (Fig. 3). Serum GLP-1 levels at $30 \mathrm{~min}$ were higher in the Aspartame group than the Control group and the levels were lowest in the Fructose group among the groups (Fig. 3). Serum triglyceride levels were higher in the Fructose than the Control and Sucralose and Aspartame lowered serum triglyceride concentrations below that of the control (Table 3 ).

Hepatic insulin signaling. Glycogen deposition in the liver was not significantly different among the groups (Fig. 4A). However, triglyceride accumulation in the liver was greater in the Aspartame group than the Control group (Fig. 4A).

In the liver the phosphorylation of Akt was lower in the Aspartame and Sucralose groups than the other groups and it was lowered by Fructose to less than the Control (Fig. 4B). Consistent with phosphorylation of Akt, the phosphorylation of GSK-3 $\beta$ also decreased in the Fructose, Aspartame and Sucralose groups more than in the Control (Fig. 4B). However, PEPCK expression

B Control Sucrose Fructose Aspartame Sucralose

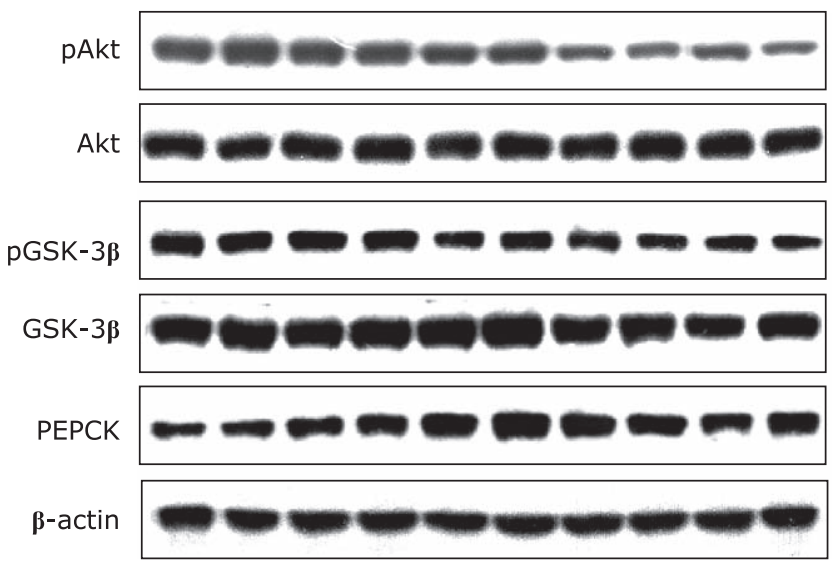

Fig. 4. Hepatic insulin signaling at the end of experiment. The ovariectomized (OVX) rats were provided with a $45 \%$ fat diet with $10 \%$ starch, sucrose, fructose, aspartame + starch and sucralose + starch for 8 weeks. After $16 \mathrm{~h}$ fasting, regular human insulin (5 U/kg body weight) was injected through their inferior vena cava. Hepatic deposition of glycogen and triglyceride was measured (A). The hepatic insulin signaling were measured with immunoblotting (B). The band intensity was measured with image analyzer (C). The bars represent means \pm SD $(n=6)$. ${ }_{a}, b, c$ The different letters on the bars represent significant differences among the groups by Tukey's test at $p<0.05$. 
increased in aspartame and Fructose group compared to the Control group (Fig. 4B). Aspartame and Sucralose were higher than the Control, but not Fructose group. Thus, other signaling influenced PEPCK expression other than insulin signaling to increase hepatic glucose output in the Fructose group.

\section{Discussion}

Aspartame and sucralose are most commonly used in processed foods, but they have some potential to have harmful effects on energy and glucose metabolism although non-nutritive sweeteners contribute little or no energy to the foods despite their sweetness. ${ }^{(26,27)}$ However, the results of metabolic studies are inconsistent, and their mechanisms are complicated. This study evaluated the acute and chronic effects of administering glucose, sucrose, fructose, aspartame or sucralose on energy metabolism, especially glucose metabolism and insulin secretion. In the acute OGTT rats administered aspartame, and to a lesser extent sucralose, had much higher peaks and AUC of insulin concentrations, even though glucose concentrations were almost the same as other groups receiving same doses of glucose. This suggested that aspartame and sucralose acutely interfered with insulin action and much more was required to normalize blood glucose concentrations. Long-term administration aspartame and sucralose caused the rats to require somewhat higher insulin levels to normalize blood glucose concentrations in OGTT without their simultaneous administration. Both aspartame and sucralose bind to a similar region of the sweet receptor. ${ }^{(28)}$ The present study demonstrated that rats fed non-nutritive sweeteners (aspartame and sucralose) were more insulin resistant than the control in an estrogen-deficient animal model, even though they had less visceral fat mass. It was associated with higher insulin secretion capacity in non-nutritive sweetener groups.

Glucose, fructose and sucrose increase insulin secretion not only by elevating serum glucose levels after their absorption but also by activating sweet taste receptor in the tongue, intestines, pancreatic $\beta$-cells and brain. ${ }^{(29)}$ The sweet taste receptors in the tongue act at the forefront of nutrient sensing to deliver the signal to prepare to maintain glucose homeostasis. ${ }^{(30)}$ Different sweeteners differently affect the taste receptor activation. Glucose triggers taste receptor signaling in the pancreatic $\beta$-cell to increase intracellular $\mathrm{Ca}^{++}$that activates phospholipase and transient receptor potential cation channel, subfamily $\mathrm{M}$, member 5 (TRPM5). ${ }^{(31)}$ The TRPM5 mediates glucose-stimulated insulin secretion by GLP-1. ${ }^{(32)}$ The common TRPM5 variants are associated with prediabetic phenotypes, and they may act as risk factors for type 2 diabetes. ${ }^{(33)}$ Fructose does not activate TRPM5, but it specifically activates taste receptor type 1 member 2 (T1R2) on pancreatic $\beta$-cells, and fructose has synergistic activity with glucose to amplify insulin release in human and mouse islets. ${ }^{(31)}$ Moreover, non-nutritive sweeteners activate T1R2 receptors in the pancreatic $\beta$-cells. Human studies demonstrate that the consumption of an oral solution of sucrose (disaccharide of glucose and fructose) can potentiate insulin release in comparison to the equimolar amounts of glucose alone, but the intake of non-nutritive sweeteners fail to modulate serum insulin or glucose levels. ${ }^{(34,35)}$ In the present study nutritive and non-nutritive sweeteners increased glucose-stimulated insulin secretion in estrogen-deficient rats, possibly by activating taste receptors in the organs.

Fructose is passively but rapidly absorbed in the intestines through glucose transporter 5 (GLUT5) and it is delivered into the liver via the portal vein. ${ }^{(36)}$ Fructose has a much lower glycemic index than glucose indicating that fructose does not increase insulin secretion as much as glucose. Fructose increases fatty acid synthesis without regulating glycolysis, and it increases the synthesis of triglycerides, which are deposited in the liver; hepatic oxidative stress and inflammation were also elevated which induce hepatic steatosis. ${ }^{(37)}$ Furthermore, the present study also showed triglyceride deposition was higher in the Fructose group than in the Control. It did not induce non-alcohol liver steatosis since the rats had a relatively low fructose diet $(11 \%)$. The fructose diet increased glycogen accumulation in OVX rats in the present study, which is possibly related to the production of fructose-1 phosphate from fructose in the liver since fructose-1-phosphate is a known inhibitor of glycogen phosphorylase that is involved in the degradation of glycogen. It might also increase gluconeogenesis from amino acids from the muscle and increased deposition of glycogen and lipid might induce hypertrophy of the liver.

In addition, increased serum acetate levels produced by gut microbiota have reported to be associated with obesity and insulin resistance by stimulating insulin secretion with activating parasympathetic nervous system. ${ }^{(38)}$ In the present study fructose intake increased visceral fat mass and insulin resistance with elevating serum acetate levels, indicating that high fructose in the diet increased production of acetate by gut microbiota. However, increased serum acetate levels did not increase insulin secretion in the Fructose group although serum glucose levels were much higher in the Fructose than other groups. As a result, fructose might suppress serum insulin levels in another way. By contrast, aspartame and sucralose did not alter serum acetate levels although they increased serum insulin levels. Therefore, high intake of fructose may facilitate the development non-alcoholic hepatic steatosis, obesity and type 2 diabetes, even though fructose has a lower glycemic index. A moderate intake of aspartame and sucralose may not exacerbate energy and glucose metabolic and gut microbiome.

Chronic intake of aspartame and sucralose also influence glucose metabolism, ${ }^{(39,40)}$ although the effect is more moderate that seen with acute administration. Non-nutritive sweeteners such as sucralose and aspartame stimulate taste receptors to signal the brain to communicate the need for pancreatic $\beta$-cell cells to prepare for insulin and GLP-1 secretion. ${ }^{(40)}$ Non-nutritive sweeteners stimulate the T1R2-T1R3 in the pancreatic $\beta$-cells to release insulin. Interestingly, chronic consumption of aspartame and sucralose increased serum insulin levels more than the control diet during OGTT. At 30 min serum GLP-1 levels were higher in the Aspartame and Sucralose groups than in the Control in the present study. Thus, sweet taste itself increased GLP-1 secretion to promote insulin. Sylvetsky et al. ${ }^{(41)}$ showed that non-nutritive sweeteners increase serum insulin concentrations and also GLP-1 secretion. Only diet soda containing aspartame elevates GLP-1 response in human subjects. ${ }^{(41)}$ Non-nutritive sweeteners may elevate GLP-1 response to release insulin from the pancreatic $\beta$ cells, but no glucose enters into the blood circulation. However, the secretion of GLP-1 and gastric inhibitory polypeptide by non-nutritive sweeteners is still controversial. ${ }^{(42,43)}$ In our acute administration of aspartame and sucralose both slightly increased glucose and insulin levels without glucose intake and in their chronic administration they elevated HOMA-IR. This could be a compensatory mechanism to protect against hypoglycemia in response to exaggerated insulin release when little or no glucose is absorbed into the blood. Increased HOMA-IR might be associated with the half-life of non-nutritive sweeteners. Saccharine and sucralose are about 13-14 h but acesulfame K and aspartame are very short. ${ }^{(41)}$ Saccharine and sucralose effect on insulin and GLP-1 secretion may be sustained for long periods. Therefore, consuming saccharine and sucralose in the fasting state may result in higher postprandial GLP-1 and insulin secretion when a meal is consumed much later, suggesting that the dosage and timing non-nutritive sweetener consumption may be important and needs to be studied in the future.

In conclusions, the chronic moderate intake of aspartame and sucralose instead of sucrose with meals may not exert harmful effects on glucose and energy metabolism in post-menopausal women, but even moderate intake of fructose intake may be 
detrimental to energy metabolism. Therefore, if the results of this animal model can be confirmed to be applicable to postmenopausal women, small amounts of aspartame and sucralose (less than $100 \mathrm{mg} /$ day) with meals might may be preferable to sweeteners such as fructose and sucrose. Even though the nonnutritive sweeteners increased insulin resistance compared to the Control. Since sucralose and aspartame have different characteristics for energy, lipid and glucose metabolism, the mixture of sucralose and aspartame should be examined to make better nonnutritive sweetener combination for the processed foods. However, caution must be observed in recommending non-nutritive sweeteners based on animal data such as these, and although we did not find the artificial sweeteners to have severe metabolic effects in this animal model, humans may react differently. Furthermore, a recent study found that the use of beverages using artificial sweeteners greatly increased the risk of stroke in women.(44) Therefore, based on our results and those of others, the best advice would be for people to avoid the use of added sweeteners as much as possible and to use glucose when a sweetener is necessary. However, human randomized clinical study is necessary to be confirmed.

\section{Author Contributions}

Authors' roles: Financial support: SP and BSK Study design: SP and JWD. Study conduct: JR, SK. Data collection: SK. Data analysis: SP, SK. Data interpretation: JR, SP, BSK, JWD. Drafting manuscript: SP. Revising manuscript content: JWD, JR, BSK. Responsibility for the integrity of the data analysis: JR and SP. Approving final version of manuscript: all authors.

\section{Acknowledgments}

This research was supported by grants from Traditional Culture Convergence Research Program through the National Research Foundation of Korea (NRF) funded by the Ministry of Science and Information and Communication Technology (NRF2016M3C1B5907049), the Korea Institute of Oriental Medicine (KNS1515290) and by the National Research Council of Science \& Technology (NST) grant by the Korea government (MSIT) (CAP-16-07-KIOM).

\section{Abbreviations}

ANOVA analysis of variance

AUC area under the curve

BMI body mass index

En\% energy percent

GLP-1 glucagon-like peptide-1

GLUT5 glucose transporter 5

GSK-3 $\beta$ glycogen synthase kinase-3 $\beta$

HOMA-IR homeostasis model assessment estimate of insulin resistance

OGTT oral glucose tolerance test

OVX ovariectomy

PEPCK phosphoenolpyruvate

REE resting energy expenditure

RQ respiratory quotient

T1R2 taste receptor type 1 member 2

$\mathrm{VCO}_{2} \quad$ carbon dioxide production

$\mathrm{VO}_{2} \quad$ oxygen consumption

\section{Conflict of Interest}

No potential conflicts of interest were disclosed.

\section{References}

1 Jung UJ, Choi MS. Obesity and its metabolic complications: the role of adipokines and the relationship between obesity, inflammation, insulin resistance, dyslipidemia and nonalcoholic fatty liver disease. Int $J \mathrm{Mol} \mathrm{Sci}$ 2014; 15: 6184-6223

2 Kanazawa M, Yoshiike N, Osaka T, Numba Y, Zimmet P, Inoue S. Criteria and classification of obesity in Japan and Asia-Oceania. World Rev Nutr Diet 2005; 94: 1-12.

3 Chan JC, Malik V, Jia W, et al. Diabetes in Asia: epidemiology, risk factors, and pathophysiology. JAMA 2009; 301: 2129-2140.

4 WHO Expert Consultation. Appropriate body-mass index for Asian populations and its implications for policy and intervention strategies. Lancet 2004; 363: $157-163$.

5 Hur YI, Park H, Kang JH, et al. Associations between sugar intake from different food sources and adiposity or cardio-metabolic risk in childhood and adolescence: the Korean Child-Adolescent Cohort Study. Nutrients 2016; 8: 20.

6 Huang M, Quddus A, Stinson L, et al. Artificially sweetened beverages, sugar-sweetened beverages, plain water, and incident diabetes mellitus in postmenopausal women: the prospective Women's Health Initiative observational study. Am J Clin Nutr 2017; 106: 614-622.

7 Ludwig DS, Peterson KE, Gortmaker SL. Relation between consumption of sugar-sweetened drinks and childhood obesity: a prospective, observational analysis. Lancet 2001; 357: 505-508.

8 Berkey CS, Rockett HR, Field AE, Gillman MW, Colditz GA. Sugar-added beverages and adolescent weight change. Obes Res 2004; 12: 778-788.

9 Trumbo PR, Rivers CR. Systematic review of the evidence for an association between sugar-sweetened beverage consumption and risk of obesity. Nutr Rev 2014; 72: 566-574.

10 Maki KC, Nieman KM, Schild AL, et al. Sugar-sweetened product consumption alters glucose homeostasis compared with dairy product consumption in men and women at risk of type 2 diabetes mellitus. J Nutr 2015; 145: 459 466.

11 Burke SJ, Batdorf HM, Martin TM, et al. Liquid sucrose consumption promotes obesity and impairs glucose tolerance without altering circulating insulin levels. Obesity (Silver Spring) 2018; 26: 1188-1196.

12 Macdonald IA. A review of recent evidence relating to sugars, insulin resistance and diabetes. Eur J Nutr 2016; 55 (Suppl 2): 17-23.

13 Malik VS, Popkin BM, Bray GA, Després JP, Willett WC, Hu FB. Sugarsweetened beverages and risk of metabolic syndrome and type 2 diabetes: a meta-analysis. Diabetes Care 2010; 33: 2477-2483.

14 Jensen T, Abdelmalek MF, Sullivan S, et al. Fructose and sugar: a major mediator of non-alcoholic fatty liver disease. J Hepatol 2018; 68: 1063-1075.

15 Raben A, Richelsen B. Artificial sweeteners: a place in the field of functional foods? Focus on obesity and related metabolic disorders. Curr Opin Clin Nutr Metab Care 2012; 15: 597-604.

16 Simon BR, Parlee SD, Learman BS, et al. Artificial sweeteners stimulate adipogenesis and suppress lipolysis independently of sweet taste receptors. $J$ Biol Chem 2013; 288: 32475-32489.

17 Steinert RE, Frey F, Töpfer A, Drewe J, Beglinger C. Effects of carbohydrate sugars and artificial sweeteners on appetite and the secretion of gastrointestinal satiety peptides. Br J Nutr 2011; 105: 1320-1328.

18 Yang HJ, Ko BS, Kwon DY, et al. Asian Elm tree inner bark prevents articular cartilage deterioration in ovariectomized obese rats with monoiodoacetateinduced osteoarthritis. Menopause 2016; 23: 197-208.

19 Kim MJ, Park JH, Kwon DY, et al. The supplementation of Korean mistletoe water extracts reduces hot flushes, dyslipidemia, hepatic steatosis, and muscle loss in ovariectomized rats. Exp Biol Med (Maywood) 2015; 240: 477-487.

20 Litwak SA, Wilson JL, Chen W, et al. Estradiol prevents fat accumulation and overcomes leptin resistance in female high-fat diet mice. Endocrinology 2014; 155: 4447-4460.

21 Park S, Kang S, Jeong DY, Jeong SY, Park JJ, Yun HS. Cyanidin and malvidin in aqueous extracts of black carrots fermented with Aspergillus oryzae prevent the impairment of energy, lipid and glucose metabolism in estrogen-deficient rats by AMPK activation. Genes Nutr 2015; 10: 455. 
22 Park S, Park CH, Jang JS. Antecedent intake of traditional Asian-style diets exacerbates pancreatic beta-cell function, growth and survival after Westernstyle diet feeding in weaning male rats. J Nutr Biochem 2006; 17: 307-318.

23 Niwa H, Ogawa Y, Kido Y, et al. The rate of lipid oxidation in septic rat models. Jpn J Surg 1989; 19: 439-445.

24 Even PC, Nadkarni NA. Indirect calorimetry in laboratory mice and rats: principles, practical considerations, interpretation and perspectives. $\mathrm{Am} \mathrm{J}$ Physiol Regul Integr Comp Physiol 2012; 303: R459-R476.

25 Yang HJ, Kwon DY, Kim MJ, et al. Red peppers with different pungencies and bioactive compounds differentially modulate energy and glucose metabolism in ovariectomized rats fed high fat diets. J Funct Food 2014; 7: 246-256.

26 Pearlman M, Obert J, Casey L. The association between artificial sweeteners and obesity. Curr Gastroenterol Rep 2017; 19: 64.

27 Suez J, Korem T, Zeevi D, et al. Artificial sweeteners induce glucose intolerance by altering the gut microbiota. Nature 2014; 514: 181-186.

28 Chan CB, Hashemi Z, Subhan FB. The impact of low and no-caloric sweeteners on glucose absorption, incretin secretion, and glucose tolerance. Appl Physiol Nutr Metab 2017; 42: 793-801.

29 Fournel A, Marlin A, Abot A, et al. Glucosensing in the gastrointestinal tract: impact on glucose metabolism. Am J Physiol Gastrointest Liver Physiol 2016; 310: G645-G658.

30 Kojima I, Nakagawa Y, Ohtsu Y, Medina A, Nagasawa M. Sweet taste-sensing receptors expressed in pancreatic $\beta$-cells: sweet molecules act as biased agonists. Endocrinol Metab (Seoul) 2014; 29: 12-19.

31 Kyriazis GA, Soundarapandian MM, Tyrberg B. Sweet taste receptor signaling in beta cells mediates fructose-induced potentiation of glucose-stimulated insulin secretion. Proc Natl Acad Sci U S A 2012; 109: E524-E532.

32 Krishnan K, Ma Z, Björklund A, Islam MS. Role of transient receptor potential melastatin-like subtype 5 channel in insulin secretion from rat $\beta$ cells. Pancreas 2014; 43: 597-604.

33 Ketterer C, Müssig K, Heni M, et al. Genetic variation within the TRPM5 locus associates with prediabetic phenotypes in subjects at increased risk for type 2 diabetes. Metabolism 2011; 60: 1325-1333.

34 Anton SD, Martin CK, Han H, et al. Effects of stevia, aspartame, and sucrose on food intake, satiety, and postprandial glucose and insulin levels. Appetite 2010; 55: 37-43.

35 Bellisle F, Drewnowski A. Intense sweeteners, energy intake and the control of body weight. Eur J Clin Nutr 2007; 61: 691-700.

36 Geidl-Flueck B, Gerber PA. Insights into the hexose liver metabolismglucose versus fructose. Nutrients 2017; 9. pii: E1026.

37 Ter Horst KW, Serlie MJ. Fructose consumption, lipogenesis, and nonalcoholic fatty liver disease. Nutrients 2017; 9. pii: E981.

38 Perry RJ, Peng L, Barry NA, et al. Acetate mediates a microbiome-brain- $\beta$ cell axis to promote metabolic syndrome. Nature 2016; 534: 213-217.

39 Romo-Romo A, Aguilar-Salinas CA, Brito-Córdova GX, Gómez Díaz RA, Vilchis Valentín D, Almeda-Valdes P. Effects of the non-nutritive sweeteners on glucose metabolism and appetite regulating hormones: systematic review of observational prospective studies and clinical trials. PLoS One 2016; 11: $\mathrm{e} 0161264$.

40 Rother KI, Conway EM, Sylvetsky AC. How non-nutritive sweeteners influence hormones and health. Trends Endocrinol Metab 2018; 29: 455-467.

41 Sylvetsky AC, Brown RJ, Blau JE, Walter M, Rother KI. Hormonal responses to non-nutritive sweeteners in water and diet soda. Nutr Metab (Lond) 2016; 13: 71 .

42 Ma J, Bellon M, Wishart JM, et al. Effect of the artificial sweetener, sucralose, on gastric emptying and incretin hormone release in healthy subjects. Am J Physiol Gastrointest Liver Physiol 2009; 296: G735-G739.

43 Shirazi-Beechey SP, Daly K, Al-Rammahi M, Moran AW, Bravo D. Role of nutrient-sensing taste 1 receptor (T1R) family members in gastrointestinal chemosensing. Br J Nutr 2014; 111 Suppl 1: S8-S15.

44 Mossavar-Rahmani Y, Kamensky V, Manson JE, et al. Artificially sweetened beverages and stroke, coronary heart disease, and all-cause mortality in the women's health initiative. Stroke 2019; 50: 555-562. 